\title{
Study on the Color of Auspicious Dolls in Yangliuqing Wooden New Year Paintings
}

\author{
Beibei Yang \\ Nankai University Binhai College \\ Tianjin, China
}

\begin{abstract}
Yangliuqing New Year painting is the first of the four Chinese New Year paintings. Among them, the auspicious doll New Year paintings are the most influential and wellknown category. The use of colors can reflect the aesthetic taste of the Chinese people and an expectation for the future. This article makes a detailed color investigation of the five most representative works in auspicious doll New Year paintings. Combined with traditional Chinese theory of color and the paint rules in folk can make analysis and summary. Explore its monochrome, color scheme and color impression. To shows a resplendent Chinese folk culture to the world and provides reference for modern design.
\end{abstract}

Keywords-Yangliuqing; New Year paintings; auspicious doll New Year painting; color

\section{INTRODUCTION}

New Year painting is a kind of Chinese painting, starting from the ancient art form "door god painting". It is one of the Chinese folk art and one of the common folk crafts as well. It was formally known as New Year paintings during Guangxu period in the Qing Dynasty. It is a unique form of painting in China, and it is also a popular art form in rural China. The New Year painting was mostly posted in the house during Chinese New Year. In order to decorate the environment, greeting the New Year and symbolize good luck in the coming New Year.

In Chinese history, there are four famous schools of New Year paintings such as Mianzhu New Year painting in Sichuan, Yangliuqing New Year painting in Tianjin, Yangjiabu New Year painting in Shandong and Taohuawu New Year painting in Jiangsu. The four schools of New Year paintings have their own characteristics. Among them, Yangliuqing New Year painting is the royal production base for New Year paintings in Qing Dynasty. It originated in Yangliuqing which in the thirty kilometers southwest of Tianjin. And the distance between Yangliuqing and Beijing is $100 \mathrm{~km}$.

Yangliuqing New Year painting has the most extensive theme, the most complex engraving, the most detailed painting and the most elegant color, so it is the most influential among the four schools of New Year paintings. The themes of Yangliuqing New Year painting include auspicious doll, maid and doll, door gods, legend and historical events, famous operas and local customs. Among them, the auspicious doll category is the most widely spread and the most influential. The auspicious doll is plump and lively. They may hold lotus flowers or embrace carps. They all looks endearing and symbolize auspiciousness and beauty. This kind of New Year paintings usually use warm and bright colors, the objects in the paintings are rendered with light color. So, the paintings look bright and clean, the color is elegant and fresh.

Therefore, the Yangliuqing paintings are one of the most representative Chinese New Year paintings especially the theme of auspicious doll. The use and composition of its color has a high research value.

\section{THE THEORETICAL BASIS OF THIS STUDY}

The theoretical basis of color investigation and analysis in this paper comes from the relevant theoretical research results which were published by the NIPPON COLOR \& DESIGN RESEARCH INSTITUTE INC. (NCD) in 1973 at the world color information conference. The color system established by NCD is based on Munsell 's theory of color. It makes the meaning of color further systematization and datamation from the image. A system that suitable for local cultural environment was constructed by using the foundations of sociology, imagology, and psychology. It is a color image system that restructures cultural genes. And it is also a color image system with universal significance the theoretical system and research method made by Munsell have been generally recognized in the field of color all over the world. This system is widely used by NCD in the fields of public utilities, environmental construction, visual communication, culture and art, commercial marketing, etc. "Color image coordinates" combine color scheme, language, environment and people into an organic ensemble. And that makes us use color just as language.

There are 120 basic colors in the system, including 10 basic hues and 12 tones. In addition, there are 10 neutral colors. In total the system has 130 basic colors. The expanded system has more hue and more nuanced hue differentiation, with a total of 1,093 colors. The color card that used to make investigation is as shown in "Fig. 1". There are four directions in the color image coordinates. The four directions respectively represent "cold" "warm" "soft" and "hard". And it has 16 basic partitions as shown in "Fig. 2", they are pretty, romantic, clear, casual, nature, elegant, chic, cool \& casual, dynamic, luxurious, wild, classic, dandy, classic \& dandy, formal \& modern. 


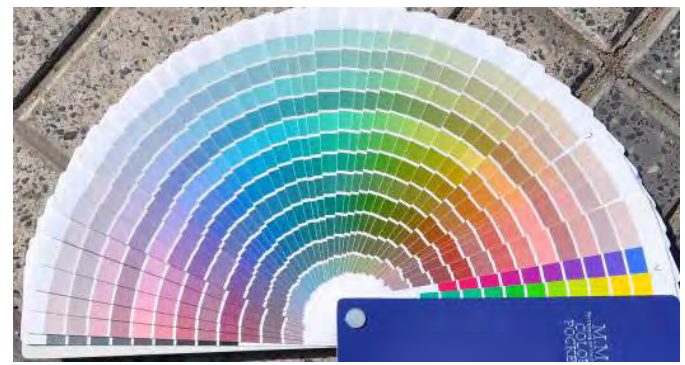

Fig. 1. Color card for survey.

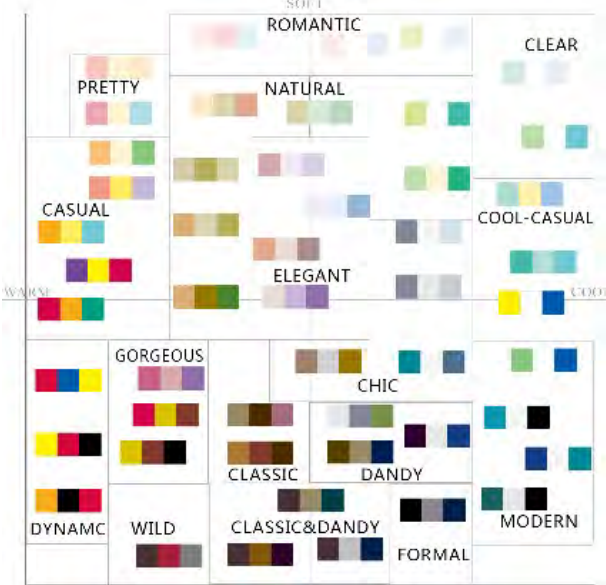

Fig. 2. NCD Color image coordinates.

\section{SurVey PROCESS AND SPECIFIC SURVEY DATA}

The auspicious doll New Year paintings are produced in large quantities every year, but it has several fixed classic styles. This article has selected the five most common styles to make color investigation and analysis. They are Live a long life, great felicity long life and posterities, be abundant forever, annual surplus and many blessing and longevity.
The way of investigation is using card to measure the color of every painting and make a data record. And then divide each painting to fill the color. In this way, some content can be analyzed through its color data and color block diagram. Such as the overall distribution of color, relations of color proportional distribution and the position of monochrome and color combination in image coordinates

\section{A. Live a Long Life}

There is a doll sitting behind a big peach as shown in "Fig. 3". And the peach is the symbol of longevity in China. So, the painting is called Live a long life. There are some other decorations beside the doll such as the pomegranate, the Buddha hand and the peach. And these three kinds of fresh fruit also represent the good meaning.

We can get some conclusion from the data of investigation. In the use of color, except the background color, the color R/V (hue: red, tone: vivid) accounts for the largest proportion, with a rate of $21 \%$. The second color in proportion is YR/Vp (hue: yellow red, tone: very pale), with a rate of $20 \%$. The third color in proportion is B/L (hue: blue, tone: light), with a rate of $15 \%$. And other colors account for less than $45 \%$ in total. From the hue distribution of the image, we can see the main color is the warm red, orange and yellow, with a proportion of $61 \%$. Analyse from the tonal aspect, bright vivid color tone accounts in a proportion of $54 \%$ in total, dark color tone accounts in a proportion of $27 \%$, and the neutral color accounts in a proportion of 3\%. From the result of the distribution of monochromatic image coordinates, we can see the distribution of the color using is extensive, covered many hues and tones. The painting has a rich image and contrasting with colors. From the result of the distribution of color image coordinates, we can see that most color distribute in the "dynamic" area of the third quadrant of the coordinates. So, the color of the image looks brilliant, dynamic and rich. 

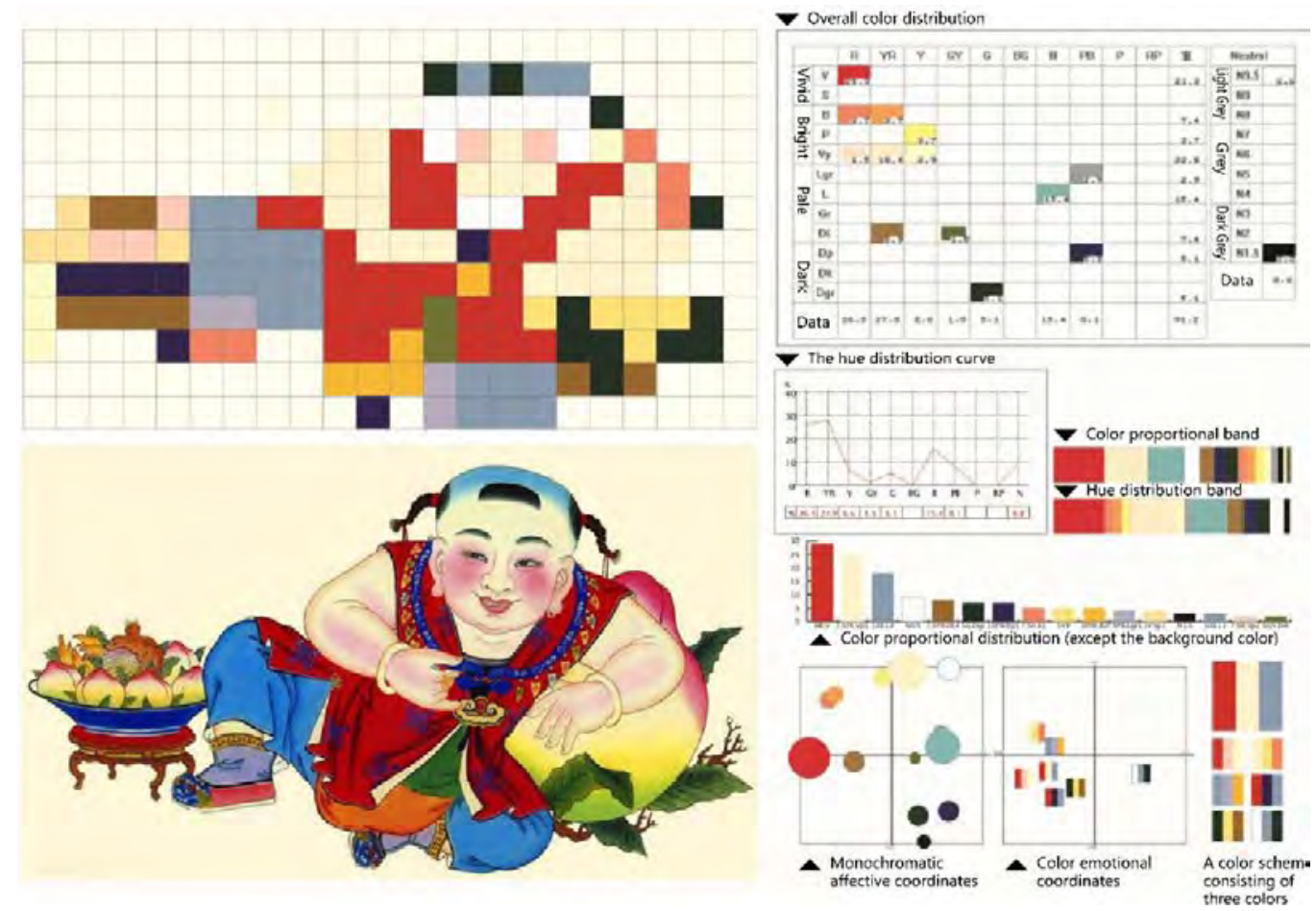

Fig. 3. Live a long life.

\section{B. Great Felicity Long Life and Posterities}

There are three dolls in the painting. Each one has a fresh fruit, such as the pomegranate, the foshou and the peach. And these three kinds of fresh fruit represent the longevity, good fortune, and posterities. The moral comes from a legend about the Yao Emperor. It called Huafengsanzhu which means congratulatory wishes for happiness, longevity and posterities. It is said that Yao comes to a place called Hua (a place name in the Xia dynasty is one of the birthplaces of Chinese civilization). The local official said to the emperor, "wish you have happiness, longevity and many posterities." Later, it became a folk saying, used to chant people or self-praise.

We can get some conclusion from the data of investigation. In the use of color, except the background color, the color YR/Vp (hue: yellow red, tone: vivid purple) accounts for the largest proportion, with a rate of $26 \%$. The second color in proportion is $\mathrm{PB} / \mathrm{P}$ (hue: purple blue, tone: pale), with a rate of $15 \%$. The third color in proportion is Y/B (hue: yellow, tone: bright), with a rate of $10 \%$. The fourth color in proportion is GY/L (hue: green yellow, tone: light), with a rate of $9 \%$. And other colors account for less than $40 \%$ in total. From the hue distribution of the image, we can see the main color is the orange and blue purple, with a proportion of $26 \%$ and $21 \%$. Analyze from the tonal aspect, bright vivid color tone accounts in a proportion of $54 \%$ in total, other color accounts in a proportion of $31 \%$.

From the result of the distribution of monochromatic image coordinates, we can see the main color in the image is warm color which in the soft color area. From the result of the distribution of color image coordinates, we can see that most color distribute in the "pretty" "romantic" area of the fourth quadrant of the coordinates. So the color of the image looks soft, childlike and free. 

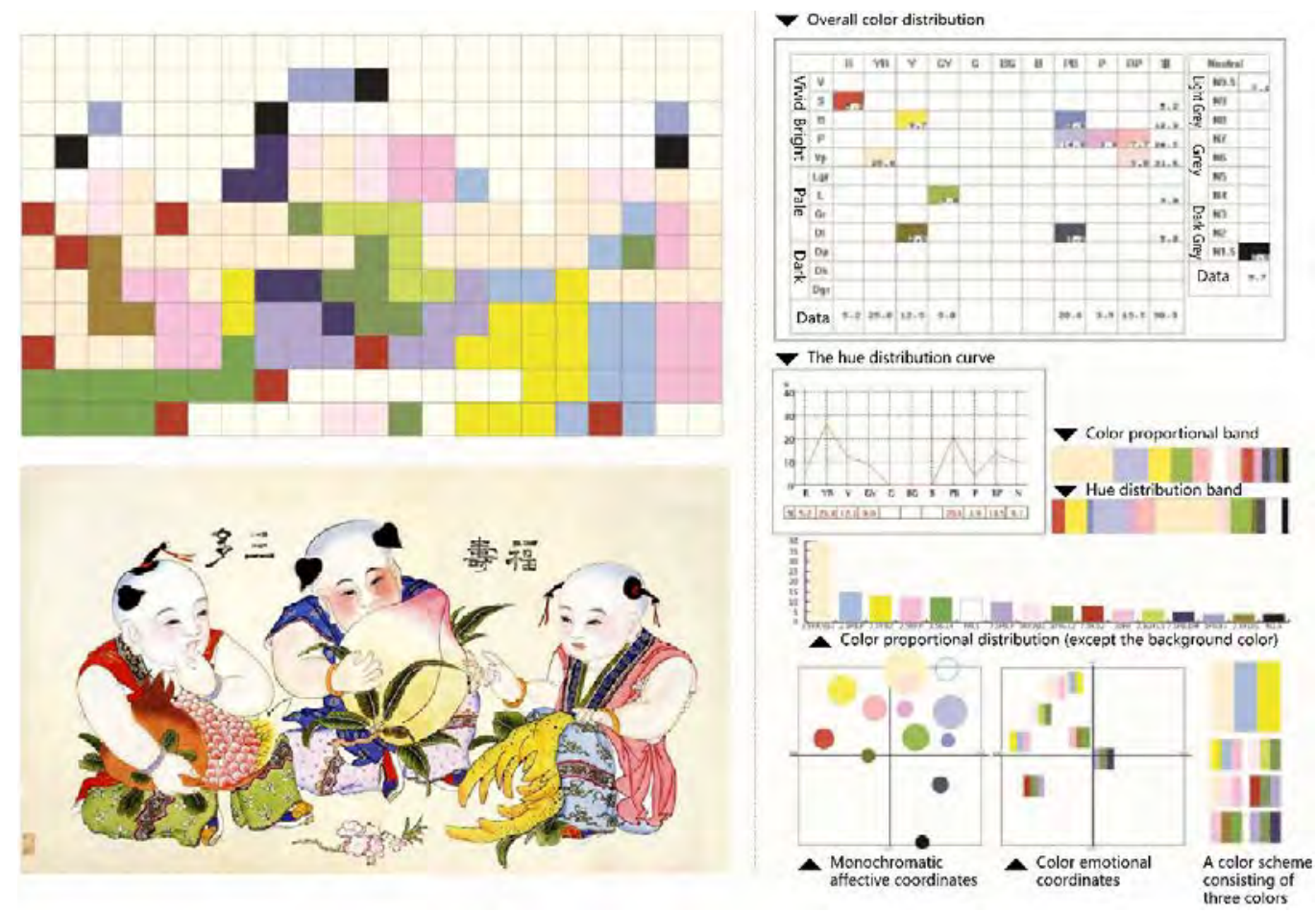

Fig. 4. Great felicity long life and posterities.

\section{A Whale Exists Thousands Years}

A whale exists thousands years which means be abundant forever is a kind of auspicious doll New Year painting. There are many images of the doll with a fish in Yangliuqing New Year painting. There is no whale in this painting, but a goldfish, as in China goldfish and whales sounds similar. The whale means the fish and thousands years is just the blessing word, means longevity.

We can get some conclusion from the data of investigation. In the use of color, except the background color, the color YR/S (hue: yellow red, tone: strong) accounts for the largest proportion, with a rate of $34 \%$. The second color in proportion is YR/Vp (hue: yellow red, tone: very pale), with a rate of $25 \%$. The third color in proportion is N1.5 (neutral colour1.5), with a rate of 9\%. And other colors account for less than $32 \%$ in total.

From the hue distribution of the image, we can see the main color is the orange, accounted for $68 \%$. Analyze from the tonal aspect, bright vivid color tone accounts for $66 \%$ in total, simple and frugal color tone accounts for $18 \%$. Other colors account for $16 \%$. From the result of the distribution of monochromatic image coordinates, we can see the main color in the image is warm orange color which in the soft color area. There are a few blue, to make color contrasting. From the result of the distribution of color image coordinates, we can see that most color distribute in the "dynamic" area of the third quadrant of the coordinates and the "casual" area of the fourth quadrant of the coordinates. So, the color of the image looks animated, dynamic and passionate. 

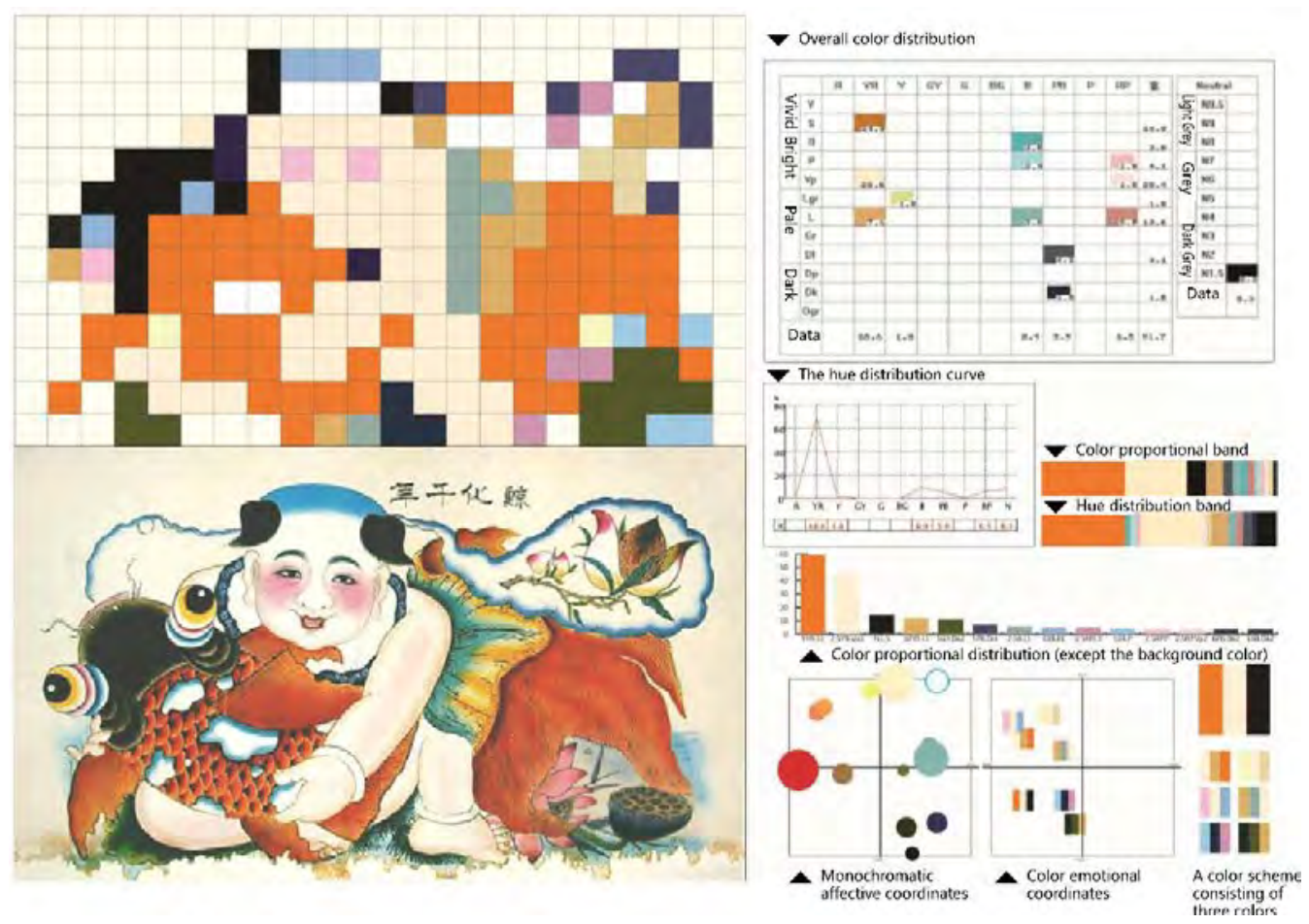

Fig. 5. A whale exists thousands years.

\section{Annual Surplus}

Annual surplus is also a kind of blessing word New Year painting. The key word in this painting is "surplus". In Chinese, surplus and fish have a similar pronunciation. So Annual surplus means you have nothing to worry and live a happy carefree life.

We can get some conclusion from the data of investigation. In the use of color, except the background color, the color P/P (hue: purple, tone: pale) accounts for the largest proportion, with a rate of $23 \%$. The second color in proportion is N9.5 (neutral colour 9.5), with a rate of $15 \%$. The third color in proportion is R/Vp (hue: red, tone: very pale), with a rate of $13 \%$. From the hue distribution of the image, we can see the main color is the cool purple and blue, accounted for $40 \%$. Analyze from the tonal aspect, bright color tone accounts for $48 \%$ in total, dark color tone accounts for $13 \%$. Other colors account for $39 \%$.

From the result of the distribution of monochromatic image coordinates, we can see the distribution of the color using is extensive, covered many hues and tones. The painting has a rich and soft image.

From the result of the distribution of color image coordinates, we can see that most color distribute in the "elegant" area of the middle quadrant of the coordinates. So, the color of the image looks supple, gentle and elegant, quiet and tranquil. 

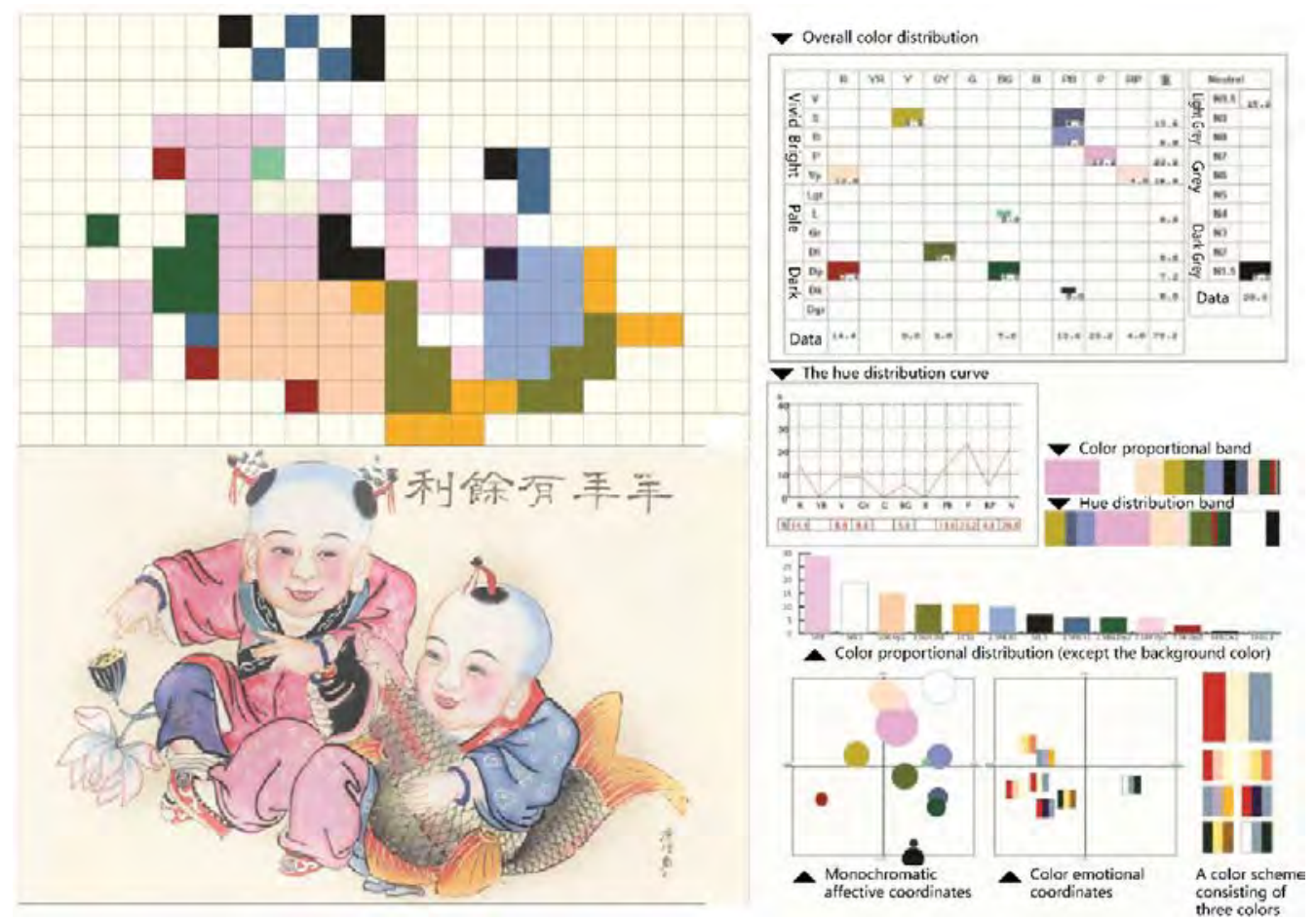

Fig. 6. Annual surplus.

\section{E. Many Blessing and Longevity}

The moral of this painting also comes from the word Huafengsanzhu which means congratulatory wishes for happiness, longevity and posterities. The image is three fresh fruits and a doll.

We can get some conclusion from the data of investigation. In the use of color, except the background color, the color P/P (hue: purple, tone: pale) accounts for the largest proportion, with a rate of $26 \%$. The second color in proportion is RP/Vp (hue: red purple, tone: very pale), with a rate of $18 \%$. The third color in proportion is N5 (neutral colour5), with a rate of $12 \%$. And other colors account for less than $44 \%$ in total. From the hue distribution of the image, we can see the main color is the purple and red purple, accounted for $44 \%$. Analyze from the tonal aspect, bright color tone accounts for $44 \%$ in total, simple and frugal color tone accounts for $22 \%$. Neutral color accounts for $20 \%$.

From the result of the distribution of monochromatic image coordinates, we can see the main color in the image is purple and green. From the result of the distribution of color image coordinates, we can see that most color distribute in the "luxurious" area of the third quadrant of the coordinates and the "casual" area of the fourth quadrant of the coordinates. So, the color of the image looks fascinating, rich and luxurious, lovely. See below "Fig. 7". 


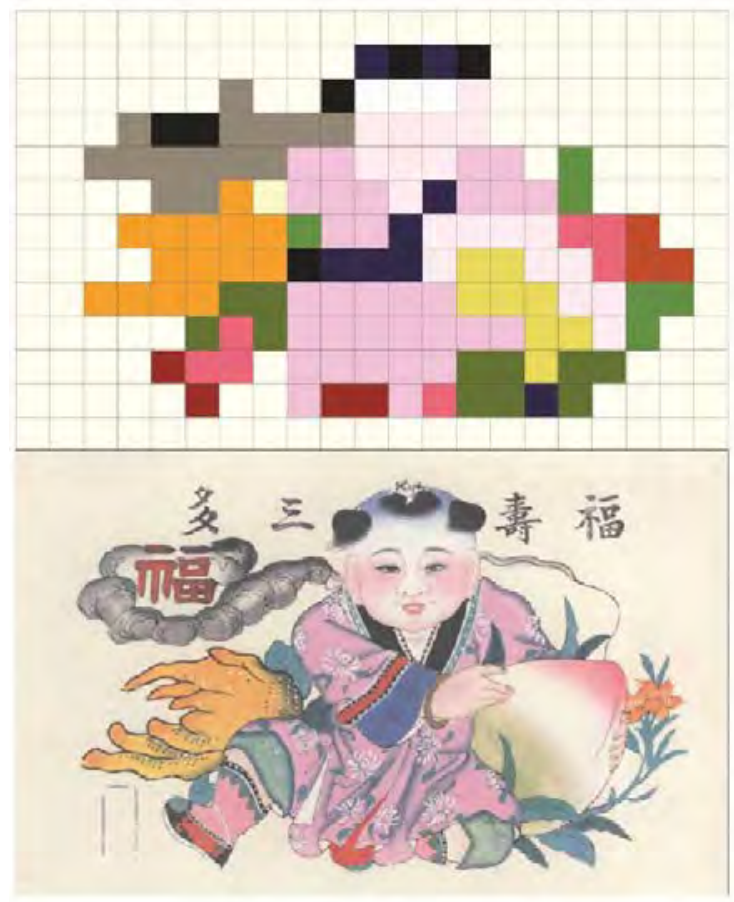

Fig. 7. Many blessing and longevity.

\section{COLOR ANALYSIS AND SUMMARY}

The main contents of Yangliuqing auspicious doll New Year painting are doll, fish, peach, pomegranates, foshou and lotus flowers. Since it is the kind of auspicious New Year painting, each element in the image has its own good moral.

The doll in the New Year painting is very important. Because in China, doll represents the child and child is the symbol of life, hope and the future. A healthy and lively child is a family's hope and spiritual support. The doll in Yangliuqing New Year painting is very lovable with its plump shape and exaggerated proportion. Doll's hair accessories, clothing is still the Ming and Qing dynasty children's clothing modeling. The color of the doll's skin is warm and elegant. The color of clothes mostly uses high chroma red, pink and blue. On the one hand it can highlight the delicate skin of the doll; on the other hand it can increase the visual impact of the picture.

Chinese people are used to bring fruit and cakes to each other as the gift during festivals. Therefore, fresh fruit often appears in auspicious New Year paintings. In New Year painting almost every fruit has its specific meaning. For example, the peach represents the longevity. It is said that it comes from a poem called Taoyao. And this poem comes from a collection of poems in Zhou Dynasty called The Book of Songs. The poem describes a scene that the peach blossom blooming and gorgeous. Since then, peach has been regarded by Chinese literati and poets as a symbol of good fortune and longevity. And people think peach is the fruit of the fairy

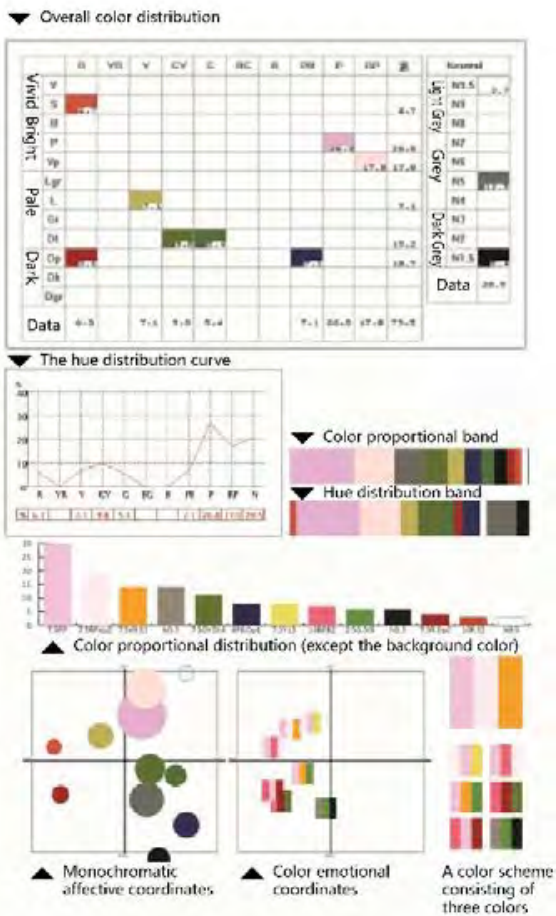

family. Life can be prolonged when you eat it. So, peach also called longevity fruit. Therefore, peach naturally becomes the symbol of longevity. Foshou is a citrus fruit. It looks like the opening fingers, so we call it foshou. In Chinese fo and happiness sound alike. So foushou is the symbol of auspicious and happiness. When the pomegranate becomes ripe it contains many pomegranate seeds. In Chinese seed and son sound alike. So, the pomegranate is the symbol of posterities. In Chinese lotus and goldfish sound like the word annual surplus. So, lotus and goldfish are the symbol of rich and abundance.

Some conclusions can be drawn by analyzing all the data. As shown in "Fig. 8", we can get some conclusion from the data of investigation. Except the influence of background color, neutral color accounts for the proportion of $13 \%$.

The color hue of YR accounts for the largest proportion, with a rate of $29 \%$. The second one is color hue of $\mathrm{R}$ accounts for the proportion of $25 \%$. And other color hues are $\mathrm{RP}, \mathrm{Y}, \mathrm{P}, \mathrm{PB}$ account for the proportion of $10 \%$ to $8 \%$. Analyze from the tonal aspect, bright color tone accounts in a proportion of $44 \%$ in total, dark color tone accounts in a proportion of $7 \%$. Pale color tone and vivid color tone are equally matched, account in a proportion of $19 \%$ and $17 \%$. From the result of the distribution of color image coordinates, we can see the color mostly distribute in the "natural" area of the fourth quadrant of the coordinates and the "luxurious" area of the third quadrant of the coordinates. So, the color makes people soft, supple and brilliant. 


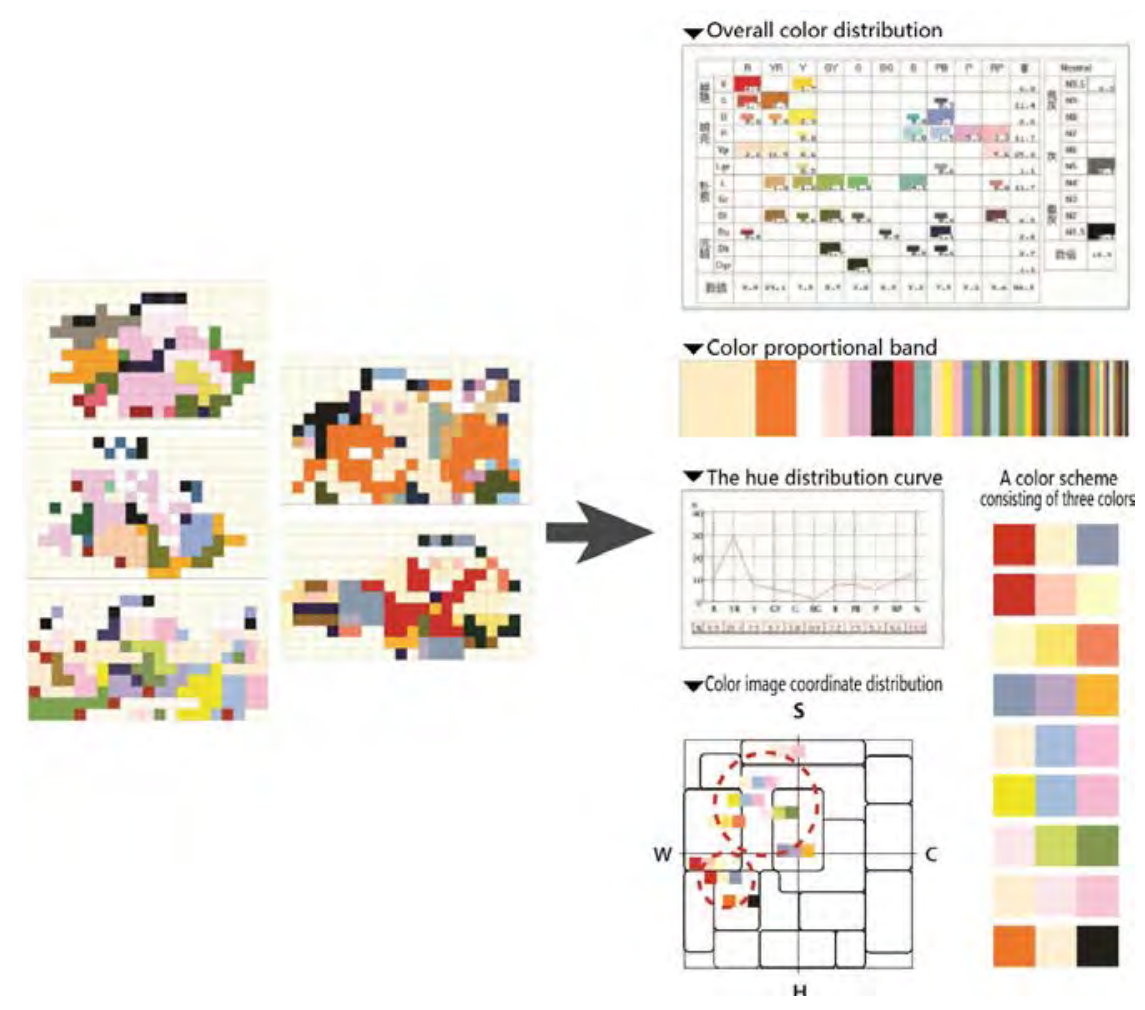

Fig. 8. Color analysis and summary.

Chinese traditional color concept is different with the western essentially. Chinese color concept comes from philosophy. It has the relationship with the five elements and yin \& yang. This is also reflected in the folk-art works, such as the use of color in Yangliuqing auspicious doll New Year paintings.

For example as shown in "Fig. 9", five elements in China correspond to five directions and five colors. And these five colors were called true color. The east corresponds to cyan,

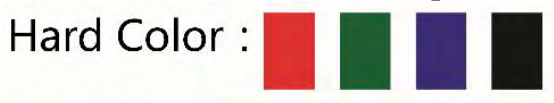

the west corresponds to white, the north corresponds to black, the south corresponds to red and the middle corresponds to yellow. Other colors are intermediate colors, such as purple, green etc. The true colors have the higher position than intermediate colors. And yellow is the most important one of the true colors. In Yangliuqing auspicious doll New Year paintings, the performance object is the doll. It means auspicious, but the color mostly using orange, pink and purple, not the true colors.
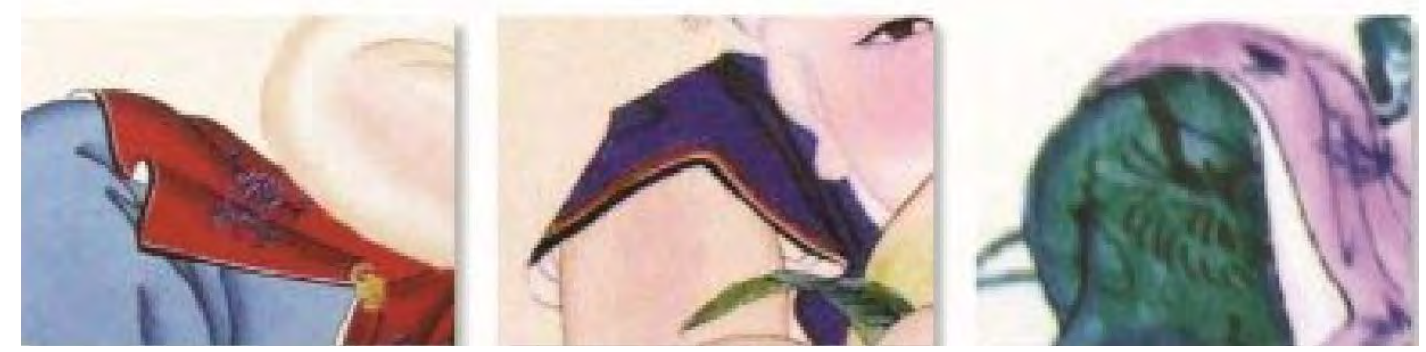

Fig. 9. Hard color and soft color

Traditional Chinese philosophy Yinyang corresponds to soft color and hard color. People usually think the soft color is light and soft and hard color is hard and heavy. But from the angle of modern chromatics, soft color is the high lightness color and the hard color is the low lightness color. There is a sentence in folk. That is soft color with hard color, make the color harmonious. It means, soft and hard colors are used next to each other will make the image looks harmonious. Such details are also common in Yangliuqing auspicious doll New Year paintings.

\section{CONCLUSION}

Yangliuqing auspicious doll New Year paintings are the treasures of Chinese folk art. In the past five hundred years, it has gradually formed its own unique style. Under the background of protecting traditional culture and skills, how 
to make the traditional art inherit and develop with more influence. It is the great difficulty and challenge that contemporary Chinese people face. Combined with the results of color analysis, traditional Chinese concept of color and the paint rules in folk can get a color atlas of Yangliuqing auspicious doll New Year painting, as shown in "Fig. 10".

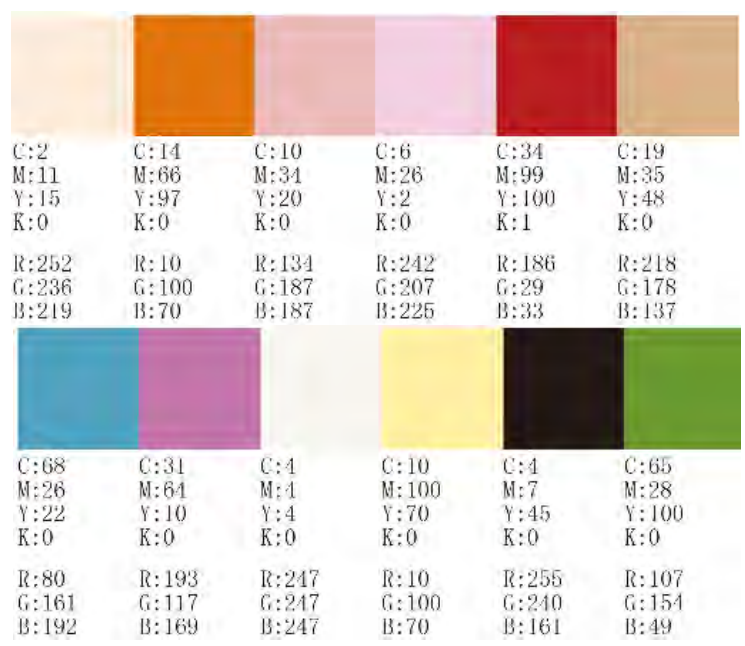

Fig. 10. Color tips.

It can not only provide references for modern design, but also show the unique charm of Chinese folk art to the world. And Yangliuqing auspicious doll New Year painting also can be recognized by the world, not only inherit in China.

\section{REFERENCES}

[1] Shigenobu Kobayashi (2006). Image color handbook [M]. Beijing: People's Fine Arts Publishing House

[2] Shigenobu Kobayashi (2006). Analysis of color psychology [M]. Beijing: People's Fine Arts Publishing House

[3] Feng Jicai (2013). Chinese New Year picture integrated Yangliuqing [M]. Beijing: Zhonghua Book Company.

[4] Han Yuxia. Yangliuqing New Year Pictures[J].China \& the World Cultural Exchange, 1993.

[5] Han Bing. A brief analysis of the color characteristics of Yangliuqing New Year paintings [J]. Art education, 2010, (9).

[6] Yang Ye. A brief analysis of the image of doll in Yangliuqing's New Year paintings [J]. Art education research, 2013, (9). 